\title{
Energy loss of protons and deuterons at low energies in Pd polycrystalline thin films
}

\author{
C. Celedón, * E. A. Sánchez, M. S. Moreno, and N. R. Arista \\ Centro Atómico Bariloche and Instituto Balseiro, San Carlos de Bariloche, RA-8400 Argentina \\ J. D. Uribe, M. Mery, J. E. Valdés, and P. Vargas \\ Laboratorio de Colisiones Atómicas, Departamento de Física, UTFSM, Valparaíso, Chile
}

(Received 7 June 2013; published 26 July 2013)

\begin{abstract}
We have investigated experimentally and by computer simulations the energy-loss distributions of low-energy $(E<10 \mathrm{keV})$ protons and deuterons transmitted through polycrystalline palladium thin films. In contrast to previous experiments on various transition metals we find that the stopping power of $\mathrm{Pd}$ is proportional to the ion velocity. Data of protons and deuterons are coincident within the experimental uncertainties, showing the absence of an isotopic effect on the stopping power of Pd in this energy range. The experimental results were analyzed and compared with Monte Carlo computer simulations and previous theoretical models. The difference in the velocity dependence of the energy loss of hydrogen ions in $\mathrm{Pd}$ with respect to other transition metals $(\mathrm{Cu}, \mathrm{Ag}$, and $\mathrm{Au}$ ) is explained by a theoretical analysis based on the properties of the $d$-electron bands of those elements.

DOI: 10.1103/PhysRevA.88.012903

PACS number(s): $34.50 . \mathrm{Bw}, 34.50 . \mathrm{Fa}$
\end{abstract}

\section{INTRODUCTION}

The physics of the interactions of ions with matter is currently a subject of great interest due to its relevance both in basic research and in numerous applications, such as health physics, radiation damage, space flights, fast semiconductor devices, and others. In a more particular context, the ability of palladium to absorb hydrogen has been an aspect of great interest for basic and applied research stimulated by technological applications in hydrogen-storage devices. The energy loss of low-energy ions is also important for ion implantation in nanoscale materials because of the current tendency of decreasing thickness and sizes in electronic devices. In a different area of research, hydrogen and its isotopes, such as deuterium and tritium, are also relevant for advanced research on nuclear fusion reactors; in this case the energy range below $10 \mathrm{keV}$ is of the greatest interest. In all of these areas, there is a need for precise information on the energy dissipation and related radiation-damage effects that these projectiles may produce after bombardment of different materials or in the electronic interactions of hydrogen with elements used in energy-storage devices.

Unlike the high-energy range, where experimental stopping power data are available for many materials, experimental work on low-energy hydrogen beams in Pd is very scarce, in particular with respect to the energy loss and energy and angular dispersion of hydrogen beams in Pd targets.

The standard energy-loss theories for low-velocity light ions in metals, which consider the target as a free-electron gas, predict a proportionality of the stopping power with the ion velocity [1-4]. This velocity dependence was experimentally confirmed for most metallic targets. However, a distinct behavior of the low-energy stopping coefficient was observed by Valdés et al. [5,6] for some metals containing $d$ electrons, such as $\mathrm{Cu}, \mathrm{Ag}$, and $\mathrm{Au}$. This effect was theoretically explained by the existence of a minimum energy transfer (threshold effect) for the excitation of $d$ electrons due to a shift in the

* On leave from Departamento de Física, UTFSM, Valparaíso, Chile. corresponding density of states of those metals with respect to the Fermi energy. This effect was further studied in more recent experiments performed at two laboratories [7-9], and on the other hand, the theoretical explanation of the origin of the effect was confirmed by more sophisticated calculations using time-dependent density-functional theory (TD-DFT) [10]. Since Pd is also a transition metal containing a complete shell of $d$ electrons and its electronic structure is similar to that of the previously mentioned elements, it is of great interest to determine whether a similar behavior is obtained for the corresponding friction coefficient at very low energies. The existing measurements for all the transition metals indicate so far similar deviations from the simple free-electron-gas prediction [5-9,11].

In this work we report the results of the experimental and computer simulation studies of the energy loss of low-energy protons and deuterons in thin self-supported Pd foils. A similar set of experiments has been performed in two laboratories, and also computer simulations have been made in order to check by different means the results of the experiments. The experimental results presented here are analyzed in the light of simulations and theoretical predictions based on DFT or alternative models and considering a more realistic density of states of Pd obtained from tight-binding linear muffin-tin orbital (TB-LMTO) calculations.

\section{EXPERIMENTAL SETUP}

Measurements were made in the Low Energy Accelerator (LEA) laboratory of the Centro Atómico Bariloche (LEA/CAB), Argentina, and the Laboratorio de Colisiones Atomicas (LCA) laboratory of the UTFSM (LCA/UTFSM), Chile, using similar experimental setups. The energy-loss measurements of protons and deuterons were performed with polycrystalline foils of palladium with several thicknesses using the transmission technique. The $\mathrm{H}^{+}$and $\mathrm{D}^{+}$beams were produced by a Colutron BK series hot-cathode ion source [12]. The ion beam was accelerated, focused, and filtered in mass and charge. The beam was deflected $18^{\circ}$ to remove the 


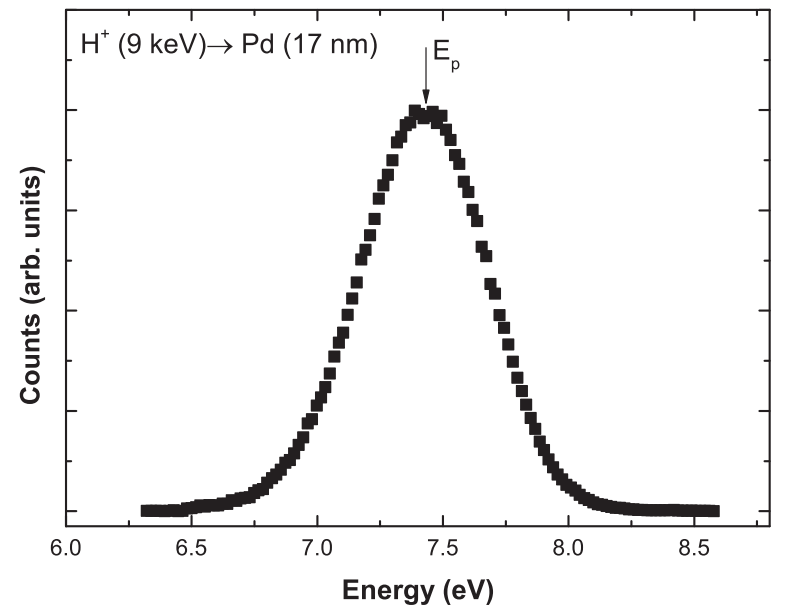

FIG. 1. Energy spectrum of 9-keV protons transmitted through a 17-nm Pd film. The arrow indicates the most probable energy of the distribution.

neutral particles before entering into a high-vacuum collision chamber. The energy-loss distributions were measured using (a) in the LEA/CAB system, an electrostatic cylindrical $\left(127^{\circ}\right)$ energy analyzer with energy and angular resolutions of $2 \%$ (FWHM) and $1.5^{\circ}$, respectively, and (b) in the LCA/UTFSM system, an electrostatic spherical $\left(90^{\circ}\right)$ energy analyzer with energy and angular resolutions of $1.3 \%$ (FWHM) and $1.5^{\circ}$, respectively. In order to minimize the elastic contribution to the energy loss, the velocity dependence of the energy loss was determined with the detector fixed in the forward direction $\left(\theta=0^{\circ}\right)$. The energy losses were determined as the difference between the incident energy $E_{0}$ and the most probable exit energy $E_{p}$ obtained from the spectra (see Fig. 1). The angular dependence of the energy loss was determined by measuring these spectra for different exit angles.

\section{A. Foil-target characterization}

The targets used were a set of Pd thin films [13], with thicknesses in the range of 16 to $18 \mathrm{~nm}$, mounted on transmission electron microscopy (TEM) grids. The film-thickness values were obtained by normalizing the measured energy loss to that calculated using the DFT friction coefficient from Ref. [14], with the $r_{s}$ value of 1.51 a.u. obtained from existing tabulations [15].

High-resolution transmission electron microscopy (HRTEM) studies were carried out in a Philips CM200 UT microscope at $200 \mathrm{kV}$ and room temperature. The HRTEM images indicated that the mean particle size was 5-10 nm. The selected-area electron diffraction (SAED) analysis showed that the Pd films were polycrystalline and contained very small traces of $\mathrm{PdO}$ phases. We estimate that these traces did not affect the present measurements beyond the experimental uncertainties.

\section{THEORETICAL BACKGROUND}

The main energy-loss mechanism for light ions in metallic targets at low and intermediate energies is the excitation of valence electrons [16], which are usually considered as a free- electron gas described as a jellium. The simplest theoretical analysis for the energy loss in metals considers the excitation of the conduction electrons by a moving ion with velocity $v$, producing a stopping force which, in the case of low energies, is proportional to the ion velocity according to

$$
d E / d x=Q\left(r_{s}\right)\langle v\rangle .
$$

Here $\langle v\rangle$ is the mean velocity of the ions in the foil,

$$
\langle v\rangle=\frac{1}{2}\left(v_{\text {in }}+v_{\text {out }}\right),
$$

and $Q\left(r_{s}\right)$ is the friction coefficient, which for a simple Fermi-gas model depends on the one-electron radius $r_{s}$ defined through the free-electron gas density $n$ by $r_{s}=(3 / 4 \pi n)^{1 / 3}$. The value of the friction coefficient $Q$ is given by [2]

$$
Q=m n v_{F} \sigma_{\mathrm{tr}},
$$

where $m$ is the electron mass, $n$ is the electron density, $v_{F}$ is the Fermi velocity, and $\sigma_{\text {tr }}$ is the momentum-transfer cross section. The properties of the electron gas are represented by the Fermi velocity $v_{F}$ and the usual $r_{s}$ parameter, which are related by $v_{F}=1.919 / r_{s}$.

Earlier models aiming to describe the energy-loss process were based on the dielectric function model (linear-response theory) [17]. This is, however, only of qualitative interest in the case of slow ions since even for protons significant nonlinear effects arise [4]. The most elaborate model to calculate the stopping coefficient $Q$ for slow ions in a free-electron gas, taking into account the nonlinear effects, is provided by the density-functional theory (DFT) [3,14]. In this approach the momentum-transfer cross section is calculated from a partialwave analysis of the scattering of target electrons in the field of the intruding ion. It should be noted that the same type of velocity proportionality is obtained from the linear and nonlinear models cited before.

As indicated in the Introduction, significant deviations from the velocity proportionality were observed for some transition metals $(\mathrm{Cu}, \mathrm{Ag}$, and $\mathrm{Au})$ at very low energies, and a theoretical explanation was proposed based on the properties of the electronic band of the $d$ electrons of those metals [6]. Hence, it is of great experimental and theoretical interest to analyze the case of palladium in detail.

\section{COMPUTER SIMULATIONS}

In order to analyze and explain the main features of the experimental results we have performed a set of computer simulations based on the Monte Carlo method. Different aspects of the method used in the Monte Carlo simulations have been described in detail in previous works $[18,19]$. The interactions between the external ion and the atoms of the solid are described in the frame of the binary-collision model. Some particular aspects of the approach are the following: For target modeling, the liquid model [20] is used; this model considers a constant traveled distance between collisions $\lambda_{0}=1 / 2 N^{-1 / 3}$, where $N$ is the atomic density of target. For surface roughness, the normal Gaussian distribution shape is used; the roughness coefficient was determined using the analysis in terms of the three-component model as in Ref. [19]. For straggling, a normal Gaussian distribution is used. The theoretical values 
of the stopping force and intrinsic straggling at low energies were obtained from the DFT calculations [14], using the same $r_{s}$ values as Ref. [15].

In these simulations we determine the trajectories of the particles traveling inside the solid and obtain the distribution of particles emerging at various observation angles as well as the energy-loss distributions of particles scattered into a given solid angle (corresponding to the detector position and size in the experiment). Multiple scattering distributions are obtained, counting the particles that emerge at a given angle $\theta$ and normalizing to the number of particles obtained in the incidence direction $\theta=0^{\circ}$. The angular dependence of the energy loss is obtained by analyzing the energy spectra of the particles emerging at different angles. Finally, the velocity dependence of the energy loss is obtained by analyzing the energy-loss spectra of particles emerging at the angle $\theta=0^{\circ}$ within the angular acceptance of the detector. Different interatomic potentials were considered in these simulations, such as Thomas-Fermi, Lenz-Jensen, Molière, and power potential [21]. The most appropriate interatomic potential for this energy range was obtained by fitting the measured angular distribution with the theoretical multiple-scattering functions, which were calculated for all these potentials using the standard multiple-scattering formulation [22], with modified values of the screening length and potential power, as described below.

\section{RESULTS AND DISCUSSION}

Figure 1 shows the energy spectrum of 9-keV protons transmitted through a 17-nm Pd foil. The data show a symmetric, almost Gaussian shape. The arrow indicates the most probable energy $E_{p}$ of the distribution.

Figure 2 shows the experimental angular distribution of 9-keV protons transmitted through the same foil, together with the normalized multiple-scattering function calculated with Lenz-Jensen, Molière, and $r^{-n}$ power potentials with screening

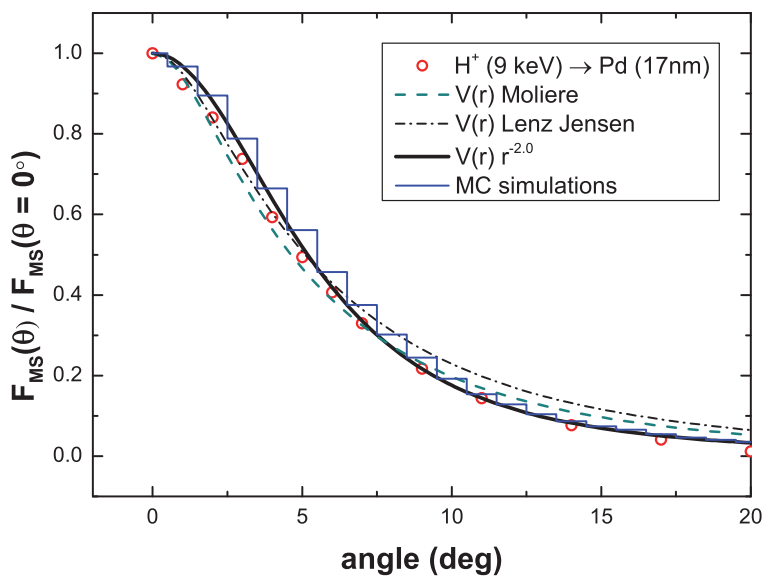

FIG. 2. (Color online) Normalized angular distribution of 9-keV protons transmitted through a thin film of $\mathrm{Pd}(17 \mathrm{~nm})$. The lines show the multiple-scattering distribution using different potentials. The dashed, dash-dotted, and solid lines correspond to the calculations considering Molière, Lenz-Jensen, and power-law potential screening functions, respectively. The step line is the result of the MC simulations

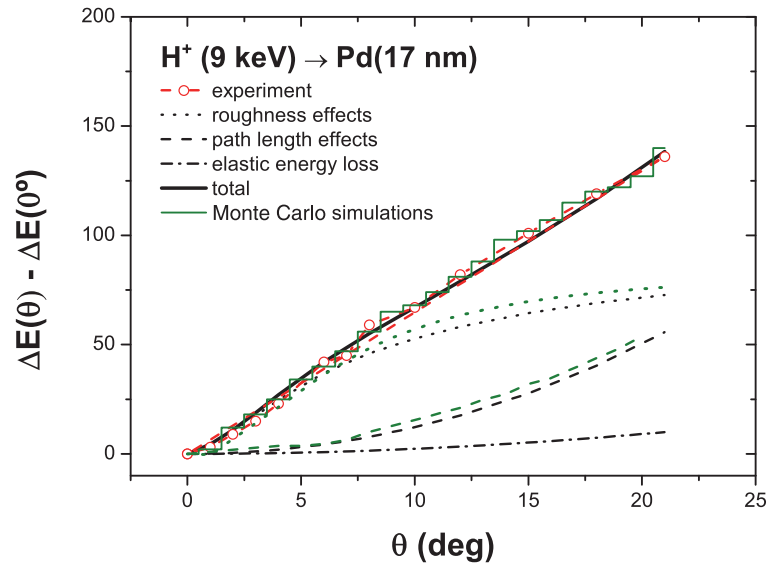

FIG. 3. (Color online) Angular dependence of the energy loss of 9-keV protons transmitted through a polycrystalline Pd film (referred to the energy loss in the forward direction). The dotted lines show the effects of foil roughness obtained from the three-component model (black) and from the MC simulation [green (gray)]. The dashed lines show the contribution of path-length enlargement obtained from the three-component model (black) and from the MC simulation [green (gray)]. The dash-dotted line shows the contribution of elastic scattering. The step line shows the MC simulations.

radii of $0.74 a_{F}, 0.68 a_{F}$, and $0.45 a_{F}$, respectively (where $a_{F}$ is the Firsov screening length [21]), using the formalism of Ref. [22]. Here we find that the $r^{-2.0}$ potential yields a better fit than the other potentials. We consider this as an empirical adjustment of the interaction potential in this energy range, as in previous theoretical analysis [23], but there is no special justification other than its fitting quality. Similar results to those shown in Fig. 2 were obtained for an energy of $7 \mathrm{keV}$, and the scaling properties predicted by theory [22] in terms of the reduced angle were verified. This potential was used in the MC simulations in order to compare with the experimental results of the angular dependence of the energy loss as a function of the observation angle.

Figure 3 shows the experimental results of the mean energy loss of ions transmitted in the forward direction as a function of the emerging angle. Here we compare the experimental results with theoretical models and simulations. On one side, we show the results of the theoretical three-component model proposed in Ref. [24]. This model allows us to separate the effects on the energy loss due to (i) the path-length enlargement (shown by the dashed line), (ii) the elastic energy-loss contribution (shown by the dash-dotted line), and (iii) the foil roughness (shown by the dotted line). The foil roughness value used in the MC simulations was $12 \%$; this value was empirically obtained as described in Ref. [24] using the multiple-scattering function shown in Fig. 2. On the other side, we show in Fig. 3, with a step line, the results of the MC simulations. As can be observed in Fig. 3, both the MC simulations and the three-component model yield a very good agreement with the experiment. The elastic contribution is found to be very small for the hydrogen-Pd case and is negligible for small observation angles; this result is a consequence of the large difference in mass between $\mathrm{H}$ and $\mathrm{Pd}$. The two main contributions to the angular dependence of the energy loss are those of path-length enlargement and foil roughness, as shown. 


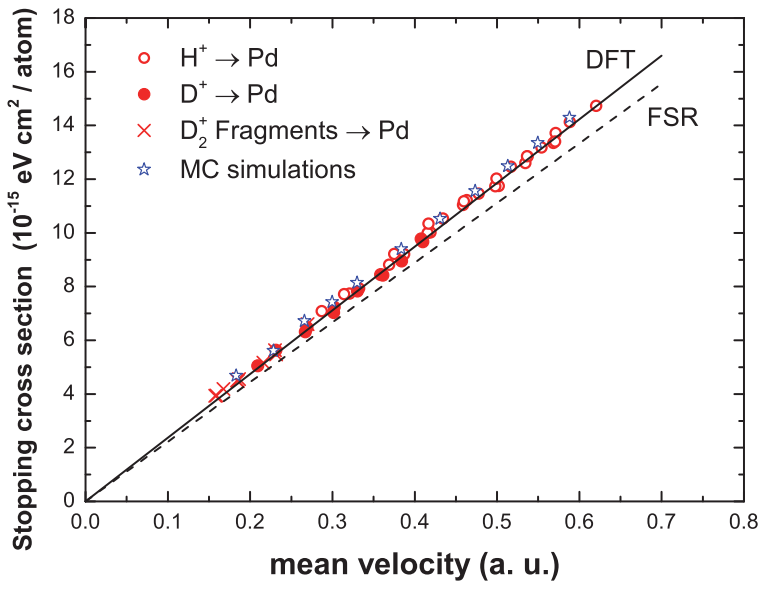

FIG. 4. (Color online) Stopping cross section for hydrogen ions in $\mathrm{Pd}$ as a function of the mean projectile velocity. Open circles show proton data; solid circles correspond to deuterons, and crosses correspond to deuteron fragments obtained from incident $\mathrm{D}_{2}{ }^{+}$ions. The high-energy value of these data was used to normalize the stopping power in this work. The solid line corresponds to the DFT calculation for a free-electron gas with $r_{s}=1.51$, and the dashed line shows the results obtained using a nonlinear model based on the Friedel sum rule.

In Figure 4 we show the projectile-velocity dependence of the stopping cross section (SCS) for $\mathrm{H}^{+}, \mathrm{D}^{+}$, and dissociation fragments (deuterons) of $\mathrm{D}_{2}{ }^{+}$incident beams transmitted in various foils of $\mathrm{Pd}$. The outgoing particles were detected in the forward direction, $\theta=0^{\circ}$ (with an angular acceptance of $1.5^{\circ}$ ) for incident particle energies of 2 to $10 \mathrm{keV}$. In this case, the measured SCS corresponds to electronic energy loss since the nuclear contribution is negligible for measurements in the forward direction. From the MC simulations we obtain a nuclear energy-loss contribution of $<1 \%$ for particles emerging in the forward direction and within the acceptance angle of the detector. Differences between the experimental data of SCS in $\mathrm{H}^{+}, \mathrm{D}^{+}$, and broken- $\mathrm{D}_{2}{ }^{+}$deuterons are not appreciated; this result allows us to state that there is no isotopic effects in the energy loss for projectiles with $Z=1$. The experimental values of SCS for protons and deuterons exhibit a linear velocity dependence in the low-velocity regime. This result is in agreement with the theoretical predictions for a free-electron gas but in contradiction to previous results for Pd where a deviation was reported [11]. The solid and dashed lines in Fig. 4 show the results of the DFT [14] and the calculations made with an alternative nonlinear model based on the Friedel sum rule [25], which gives very close results.

Figure 5 shows the present results for the friction coefficient $Q$ [defined by Eqs. (1)-(3)] for protons and deuterons in Pd. The constant value obtained here is similar to the behavior previously observed for carbon [26] and differs from the results obtained for other transition metals $(\mathrm{Cu}, \mathrm{Ag}$, and $\mathrm{Au})$ where a drop in the $Q$ values at low speeds (below 0.3 a.u.) was observed [5-9]. This difference in the behavior of the lowenergy stopping power for different transition metals is very striking and requires a theoretical explanation.

As already noticed in the Introduction, the stopping power of transition metals in the low-velocity range is strongly linked to the behavior of the $d$-electron bands of these elements.

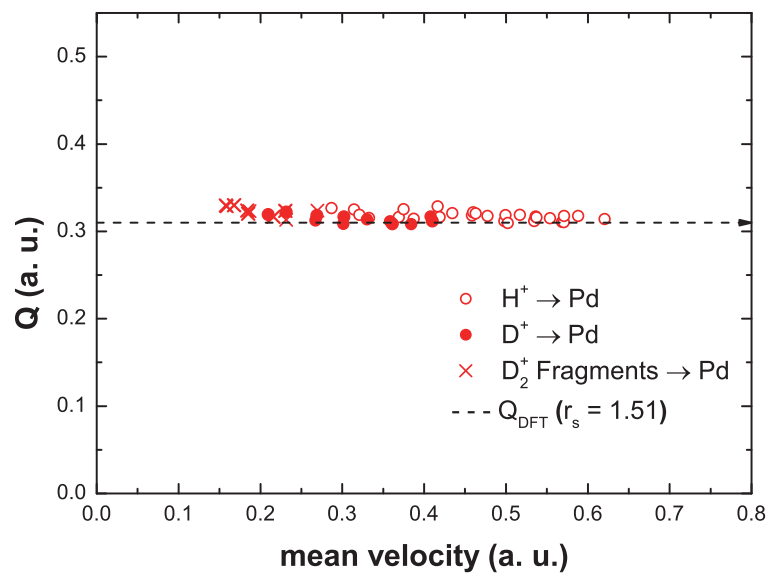

FIG. 5. (Color online) Experimental electronic friction coefficient for $\mathrm{H}^{+}, \mathrm{D}^{+}$and $\mathrm{D}_{2}{ }^{+}$fragments in $\mathrm{Pd}$ as a function of the mean projectile velocity. The dashed line represents the DFT calculations with $r_{s}=1.51$.

The properties of these electrons are well characterized by the corresponding densities of states (DOS). In Fig. 6 we show and compare the DOS for $\mathrm{Ag}$ and $\mathrm{Pd}$, calculated by one of us (PV) using TB-LMTO methods [27]. The difference in the $d$-electron bands of $\mathrm{Pd}$ and $\mathrm{Ag}$ is very clear: we see that the upper edge of the $d$-electron band of $\mathrm{Ag}$ is shifted from the Fermi level by several $\mathrm{eV}$, producing a threshold effect for the excitation of these electrons, while the $d$-electron band of Pd crosses that level. Similar differences are observed by comparing the $d$-electron bands of $\mathrm{Cu}$ and $\mathrm{Au}$ with that of $\mathrm{Pd}$. As stated in Refs. [5,6], the threshold effects in $\mathrm{Cu}, \mathrm{Ag}$, and Au comes from the presence of a "band gap" between their $d$ bands and the Fermi level. In the case of Pd this effect does not arise, so we may expect a simple metallic-like behavior, different from the other transition metals. This provides a physical explanation of the constancy in the friction coefficient observed experimentally at low energies and the absence of a threshold effect in the stopping power of $\mathrm{Pd}$.

We finally observe that the present analysis, based on the standard DFT formulation [14], reproduces fairly well

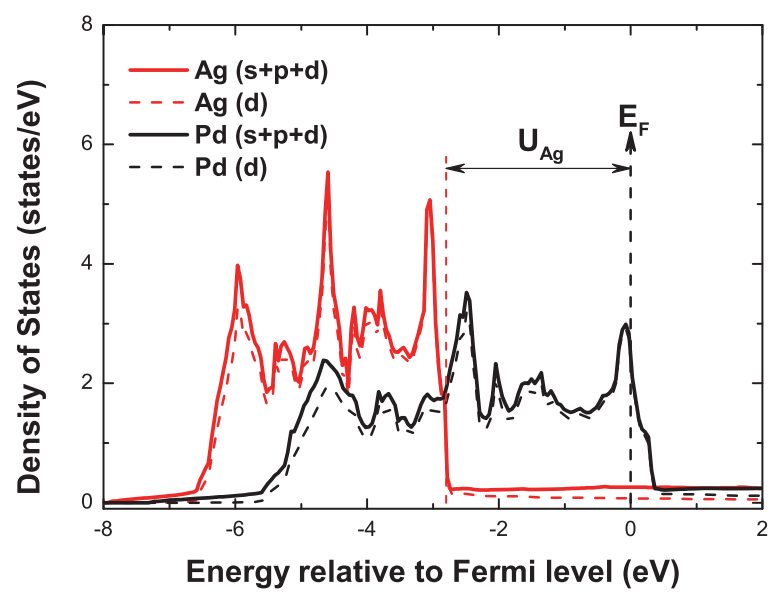

FIG. 6. (Color online) Density of states of Pd and Ag, including the contributions of $s, p$, and $d$ electrons. In the case of $\mathrm{Ag}$, the threshold energy $U$ is also indicated 
the experimental results. It may be expected that $a b$ initio calculations based on time-dependent DFT methods [10] may provide additional physical insight on this phenomenon.

\section{CONCLUSIONS}

The interaction of $\mathrm{H}^{+}$and $\mathrm{D}^{+}$with polycrystalline $\mathrm{Pd}$ was investigated by the transmission technique in the low-energy range, from 2 to $10 \mathrm{keV}$, using very thin $(16-18 \mathrm{~nm})$ foils. The angular and the energy-loss distributions and the velocity dependence of the stopping cross section were experimentally determined and compared with computer simulations based on theoretical models.

The multiple-scattering formalism of Ref. [22] yields a good agreement with the measurements of angular distributions using a power-law potential, which yields a better fit than the Molière and Lenz-Jensen potentials in this low-energy range.

The angular dependence of the energy loss can be accounted for by a previous three-component model [24], which includes the effects of path-length enlargement, elastic scattering, and surface roughness of the sample.

Measurements of the energy loss in the forward direction (close to zero-scattering angle) provided direct information on the electronic energy loss, showing no isotopic effects due to the extremely small contribution of elastic energy losses at small angles. The measurements of the electronic energy loss in the forward direction show a velocity-proportional dependence, without any evidence of the threshold effects that were observed in other transition metals.

The absence of threshold effects in Pd may be explained by the particular properties of its $d$-electron band which crosses the Fermi level, in contrast to other transition metals such as $\mathrm{Cu}, \mathrm{Ag}$, and $\mathrm{Au}$.

The velocity proportionality in $\mathrm{Pd}$, characteristic of simple metals, can be described by the standard DFT model [14] considering the $d$ electrons as free electrons described by a jellium approximation. It may be expected that these results may stimulate further theoretical studies on the dynamical response of $d$ electrons in transition metals based on ab initio methods.

\section{ACKNOWLEDGMENTS}

The authors are grateful to G. H. Lantschner for many useful comments. This work was supported in part by ANPCYT and Universidad Nacional de Cuyo (Argentina) and FONDECYT, USM-DGIP, and CEDENNA (Chile).
[1] J. Lindhard, Mat. Fys. Medd. K. Dan. Vidensk. Selsk. 28, 1 (1954).

[2] T. L. Ferrell and R. H. Ritchie, Phys. Rev. B 16, 115 (1977).

[3] P. M. Echenique, R. M. Nieminen, and R. H. Ritchie, Solid State Commun. 37, 779 (1981).

[4] A. Mann and W. Brandt, Phys. Rev. B 24, 4999 (1981).

[5] J. E. Valdés, G. Martínez Tamayo, G. H. Lantschner, J. C. Eckardt, and N. R. Arista, Nucl. Instrum. Methods Phys. Res., Sect. B 73, 313 (1993).

[6] J. E. Valds, J. C. Eckardt, G. H. Lantschner, and N. R. Arista, Phys. Rev. A 49, 1083 (1994).

[7] E. D. Cantero, G. H. Lantschner, J. C. Eckardt, and N. R. Arista, Phys. Rev. A 80, 032904 (2009).

[8] S. N. Markin, D. Primetzhofer, S. Prusa, M. Brunmayr, G. Kowarik, F. Aumayr, and P. Bauer, Phys. Rev. B 78, 195122 (2008).

[9] S. N. Markin, D. Primetzhofer, M. Spitz, and P. Bauer, Phys. Rev. B 80, 205105 (2009).

[10] M. A. Zeb, J. Kohanoff, D. Sanchez-Portal, A. Arnau, J. I. Juaristi, and E. Artacho, Phys. Rev. Lett. 108, 225504 (2012).

[11] J. E. Valdés, C. Agurto, F. Ortega, P. Vargas, R. Labbé, and N. R. Arista, Nucl. Instrum. Methods Phys. Res., Sect. B 164-165, 268 (2000).

[12] Colutron Research Corporation, Boulder, Colorado, USA, http://www.colutron.com

[13] Ted Pella Inc., Redding, California, USA, http://www.tedpella.com
[14] M. J. Puska and R. M. Nieminen, Phys. Rev. B 27, 6121 (1983).

[15] D. Isaacson, Radiation and Solid State Laboratory, New York University, Doc. No. 02698 (National Auxiliary Publication Service, New York, 1975).

[16] M. A. Kumakhov and F. F. Komarov, Energy Loss and Ion Ranges in Solids (Gordon and Breach, New York, 1981).

[17] J. Lindhard and A. Winther, Mat. Fys. Medd. K. Dan. Vidensk. Selsk. 34, 1 (1964).

[18] W. Möller, G. Pospiech, and G. Schrieder, Nucl. Instrum. Methods 130, 265 (1975).

[19] M. Famá, J. C. Eckardt, G. H. Lantschner, and N. R. Arista, Phys. Rev. A 62, 062901 (2000).

[20] W. Eckstein, Computer Simulation of Ion-Solid Interactions (Springer, Berlin, 1991).

[21] M. Nastasi, J. W. Mayer, and J. K. Hirvonen, Ion-Solid Interactions (Cambridge University Press, Cambridge, 1996).

[22] P. Sigmund and K. B. Winterbon, Nucl. Instrum. Methods 119, 541 (1974).

[23] J. Lindhard, V. Nielson, and M. Scharff, Mat. Fys. Medd. K. Dan. Vidensk. Selsk. 36, 10 (1968).

[24] M. Famá, G. H. Lantschner, J. C. Eckardt, C. D. Denton, and N. R. Arista, Nucl. Instrum. Methods Phys. Res., Sect. B 241, 164 (2000).

[25] A. F. Lifschitz and N. R. Arista, Phys. Rev. A 57, 200 (1998).

[26] E. D. Cantero, G. H. Lantschner, and N. R. Arista, Eur. Phys. J. D 65, 397 (2011).

[27] O. K. Andersen and O. Jepsen, Phys. Rev. Lett. 53, 2571 (1984). 